\title{
The Affection of Exponential Growth on Scale-free Network
}

\author{
Bei-You LI ${ }^{1, a,{ }^{*},}$ Tian-Fan SONG ${ }^{2, b}$, Rui-Ling BIAN ${ }^{3, c}$ \\ ${ }^{1}$ School of Finance, Qilu University of Technology, Jinan, China \\ ${ }^{2}$ School of Finance, Shandong University of Finance and Economics, Jinan, China \\ ${ }^{3}$ School of Mathematical Sciences, Jinan University, Jinan, China \\ a579579@qq.com, b110188951@qq.com, Br10415@163.com \\ ${ }^{*}$ Corresponding author
}

Keywords: Local world, Power-law distribution, Poisson procedure, Mean-field theory.

\begin{abstract}
The combination of growth and preferential attachment is responsible for the power-law distribution of vertices' degree in random networks; however there are many kinds of growing ways of real networks. Based on preferential attachment and exponential growth, the paper presents the analytical results which the vertices' degree of scale-free network follows power-law distribution $p k \sim k^{-r}$ $(r=2 \beta+1)$ and parameter $\beta$ satisfies $0.5<\beta \leq 1$. At same time we find that the preferential attachment is taken place in a dynamic local world and the size of the dynamic local world is in direct proportion to the size of whole networks. The paper also gives the analytical results of no-preferential attachment and exponential growth on random networks. At last, computer simulated results of the model illustrate these analytical results.
\end{abstract}

\section{Introduction}

Recently, there has been a considerable interest in the growth properties of random networks, such as WWW, the electrical distribution system and social networks. These networks all have very different physical forms, with different definition for their nodes and links. However, they appear to display considerable topological similarity, have connectivity distributions which behave as power law [1-14].

Models of growing random networks were first introduced by Barabási and Albert (B\&A) [1-3]. In B\&Amodel, network grows by addition of new nodes and new vertices preferentially attach to highly connected nodes. All vertices increase their degree following a power-law time dependence with the same dynamic exponent, $k \sim t^{1 / 2}$. So networks can self-organize into a scale free state. As a consequence, the oldest vertices have the highest number of links, since they had the longest lifetime to accumulate them.

A common characteristic of many large complex networks is that the connective degree distribution of vertices follows power law[1-8]. The connective probability $\mathrm{P}(\mathrm{k})$ that a vertex in the network is connected to $\mathrm{k}$ other vertices decays as a power law, following $\mathrm{P}(\mathrm{k}) \sim \mathrm{k}-\mathrm{r}[1-5]$. $\mathrm{B} \& \mathrm{~A}$ present the power law with $\mathrm{r}=3$ using mean-field theory[1]. They demonstrated that the combination of growth and preferential attachment is ultimately responsible for the scale-free distribution and power-law scaling. The growth in $\mathrm{B} \& A$ model is that the network begins from a few vertices $(\mathrm{m} 0)$ and a new vertex with $\mathrm{m}$ edges $(\mathrm{m} \leq \mathrm{m} 0)$ is added to the network at each step time. So it is uniform growth.

However, many studies on real networks demonstrate that the power-law distribution of vertex degree in many scale-free network is not the only one whose $r=3$ (see Tab.1)[4-5]. The exponent of power law distribution always satisfies $2<r \leq 3$. We consider that different network has different speed of evolution.

Some events take place randomly and have certain independence, such as, the coming of telephone call in telephone network, the appearing of new page in WWW, etc. The interval time between two events is not equal to other. So, exponential distribution which followed by the interval time between two vertices is a suitable method which describes the random phenomena. 
Table 1 Some scale-free networks[4] (abridged)

\begin{tabular}{|c|c|c|c|}
\hline Networks & size & type & rout \\
\hline Co-authors, neuro & 209293 & undirected & 2.1 \\
\hline Internet domain & 4389 & undirected & 2.2 \\
\hline Movie actors & 212250 & undirected & 2.3 \\
\hline Co-authors, maths & 70975 & undirected & 2.5 \\
\hline WWW nd.edu & 325729 & directed & 2.1 \\
\hline Internet router & 150000 & undirected & 2.4 \\
\hline WWW Altavista & $2 * 108$ & directed & 2.72 \\
\hline Words, co-occurrence & 460902 & undirected & 2.7 \\
\hline Words, synonyms & 22311 & undirected & 2.8 \\
\hline
\end{tabular}

Li \& Chen present a evolution model of local world in 2003[6]. After given a fixed size M of local world, the $\mathrm{M}$ vertices are selected randomly from the existing network firstly, and the new vertex attached preferentially to $m$ vertices which are selected the M vertices of local world. Extremely, the "local world" of B\&A model is the whole network.

In the paper, we will give the effect of network evolution speed $\beta$ on power-law exponent based on mean-field theory. At the same time, the dynamic local world will comes from exponential growth of network, whose size is in direct proportion to the size of whole network.

\section{Exponential Growth and Preferential Attachment}

The model of B\&A demonstrates the effect of growth and preferential attachment on power-law distribution $[1,4,5]$. The vertex is added to network at the same interval time in B\&A model, and the probability that a new vertex will be connected to vertex i depends on the connectivity ki of that vertex.

Here, we change the vertex growing interval time from unchanged to following exponential distribution, and keep the preferential attachment. The new model is as following:

(1) Exponential growth: starting with a small number (n0) of vertices and a small number (m0) of edges. In interval time $\mathrm{t}$ a new vertex with $\mathrm{m}(\mathrm{m} \leq \mathrm{n} 0)$ edges is added the network. The $\mathrm{m}$ edges will be connected to the vertices already present in the network. The interval time $t$ follows exponential distribution with parameter $\beta$.

(2) Preferential attachment: when choosing the vertices to which the new vertex connects, we assume that the probability $\pi k_{i}$ that a new vertex will be connected to vertex i depends on the connectivity $k_{i}$ of that vertex, such that

$$
\pi k_{i}=\frac{k_{i}}{\sum_{j} k_{j}}
$$

Because the interval time $\mathrm{t}$ at which each vertex appears follows the exponential distribution $f x=\beta e^{-\beta x}, \beta>0, x>0$, the counting process $N t$ at the time interval $[0, t]$ follows the Poisson distribution $P$ N $t=k=\frac{\beta t^{k}}{k !} e^{-\beta t}$. Then, the number $\mathrm{n}$ of vertices which appeared at each unit time follows Poisson distribution $P \quad n=k=\frac{\beta^{k}}{k !} e^{-\beta}$.

In a unit time, the uniform probability which a new vertex connects to one of the already existed vertices in network is $p=\frac{1}{n_{0}+\beta t}$. As $t \rightarrow \infty, p=\frac{1}{m_{0}+\beta t} \rightarrow 0$ can satisfy the condition of binomial 
distribution approaching to Poisson distribution: larger N, smaller p. So we can obtain that $\beta=N p$, so $N=\beta n_{0}+\beta t$.

Because of the approach from binomial distribution to Poisson distribution, we can obtain that $\beta=N C_{N}^{m} p^{m} 1-p^{N-m}$, i.e., the events which select $\mathrm{m}$ vertices from $\mathrm{N}$ vertices connected to new vertex are response to $\mathrm{m}$ times independent Bernoulli experiment. Here, $N=\beta\left(n_{0}+\beta t\right)$ is the number of local world vertices which are selected from the whole $n_{0}+\beta t$ vertices. Then we can obtain that $N=\beta n_{0}+\beta t \leq n_{0}+\beta t$. At last, we can obtain that

$$
\beta \leq 1
$$

We calculate analytically the probability $p k$ using mean-field theory, so that the scaling exponent $\mathrm{r}$ could be determined exactly.

Assumed that $\mathrm{k}$ is continuous, we can write for a vertex $\mathrm{i}$

$$
\frac{\partial k_{i}}{\partial t}=A \pi k_{i}=A \frac{k_{i}}{\sum_{j} k_{j}}
$$

At the moment $\mathrm{t}$, there are $\beta t$ vertices which have been added to network averagely. So the model becomes a random network in which there are $N=n_{0}+\beta t$ vertices and $M=m_{0}+m \beta t$ edges.

Then, $\sum_{j} k_{j}=2 m \beta t$. At the interval time $\Delta t$ which there is only one vertex added to network the change in connectivity is $\Delta k=m$, we obtain that $\mathrm{A}=\mathrm{m}$, leading to

$$
\frac{\partial k_{i}}{\partial t}=\frac{k_{i}}{2 \beta t}
$$

The initial condition of equation (4) is that vertex $\mathrm{i}$ was added to the network at time $t_{i}$ with connectivity $k_{i}\left(t_{i}\right)=m$. We can obtain that the solution of equation is

$$
k_{i} t=m \times\left(\frac{t}{t_{i}}\right)^{\frac{1}{2 \beta}}
$$

The exponent $\frac{1}{2 \beta}$ is bounded, i.e. $0<\frac{1}{2 \beta}<1$ because a node always increase the number of links in time $\left(\frac{1}{2 \beta}>0\right)$ and $k_{i} t$ cannot increase faster than $\mathrm{t}\left(\frac{1}{2 \beta}<1\right)$. So the parameter $\beta$ satisfies

$$
\beta>0.5
$$

From inequation (2) and (6), we can obtain that

$$
0.5<\beta \leq 1
$$

The probability that a vertex has a connectivity $k_{i} t, P k_{i} t<k$, can be written as

$$
P k_{i} t<k=P\left(t_{i}>\left(\frac{m}{k}\right)^{2 \beta} \cdot t\right)
$$

Because of the assumption of network growth, the probability density of $t_{i}$ is 


$$
p_{i} t_{i}=\beta e^{-\beta t_{i}}
$$

So we can obtain that

$$
P\left(t_{i}>\left(\frac{m}{k}\right)^{2 \beta} \cdot t\right)=1-P\left(t_{i} \leq\left(\frac{m}{k}\right)^{2 \beta} \cdot t\right)=e^{-\beta t\left(\frac{m}{k}\right)^{2 \beta}}
$$

The probability density for $p k$ can be obtained using

$$
p k=\frac{\partial P k_{i} t<k}{\partial k}=e^{-\beta t\left(\frac{m}{k}\right)^{2 \beta}} \cdot 2 \beta^{2} t m^{2 \beta} \cdot \frac{1}{k^{2 \beta+1}}
$$

Through the Taylor series, we can obtain that

$$
e^{-\beta t\left(\frac{m}{k}\right)^{2 \beta}}=1-\beta t\left(\frac{m}{k}\right)^{2 \beta}+\frac{1}{2 !} \beta^{2} t^{2} \cdot\left(\frac{m}{k}\right)^{4 \beta}-\ldots \ldots
$$

Because of $k>m$, we can obtain that

$$
p k \approx 2 \beta^{2} t \cdot m^{2 \beta} \cdot \frac{1}{k^{2 \beta+1}}
$$

The exponent of power-law distribution is $r=2 \beta+1$, where $0.5<\beta \leq 1$. So we can obtain that $2<r \leq 3$.

\section{Exponential Growth But Non-preferential Attachment}

B\&A study two compare models respectively: model A with uniform growth but non-preferential attachment, model B with preferential attachment but no-growing[1]. For testing and verifying the effect of exponential growth on network, we also present the analytical result of the model with exponential growth but non-preferential attachment.

The assumption for model with exponential growth but non-preferential attachment is as following:

(I) Exponential growth: starting with a small number $(\mathrm{n} 0)$ of vertices and a small number $(\mathrm{m} 0)$ of edges. In interval time $t$ a new vertex with $\mathrm{m}(\mathrm{m} \leq \mathrm{n} 0)$ edges is added the network. The $\mathrm{m}$ edges will be connected to the vertices already present in the network. The interval time t follows exponential distribution with parameter $\beta$.

(II) Uniform attachment: we assume that the new vertex connects with equal probability to the vertices already exist in the network. At the moment $\mathrm{t}$, there are $\beta t$ vertices which have been added to network averagely. So, $\pi k_{i}=\frac{1}{n_{0}+\beta t-1}$, independent of $k_{i}$.

By means of mean-field theory, we can obtain that

$$
\frac{\partial k_{i}}{\partial t}=A \pi k_{i}=\frac{A}{m_{0}+\beta t-1}
$$

At the interval time $\Delta t, \Delta k=m$ then $A=m$.

The initial condition of equation (13) is that vertex $\mathrm{i}$ was added to the network at time $t_{i}$ with connectivity $k_{i}\left(t_{i}\right)=m$. We can obtain that the solution of equation is

$$
k_{i} t=m\left[\ln \left(\frac{m_{0}+\beta t-1}{m_{0}+\beta t_{i}-1}\right)+1\right]
$$


The probability that a vertex has a connectivity $k_{i} t$ smaller than $k, P k_{i} t<k$, can be written as

$P k_{i} t<t=P\left(t_{i}>\frac{1}{\beta}\left[m_{0}+\beta t-1 e^{1-\frac{k}{m}}-m_{0}+1\right]\right)$

Because of the assumption of network growth, the probability density of $t_{i}$ is

$$
p_{i} t_{i}=\beta e^{-\beta t_{i}}
$$

So we can obtain that

$$
P\left(t_{i}>\frac{1}{\beta}\left[m_{0}+\beta t-1 e^{1-\frac{k}{m}}-m_{0}+1\right]\right)=e^{\left.-\mid m_{0}+\beta t-1 e^{1-\frac{k}{m}}-m_{0}+1\right]}
$$

Calculating the derivative of the former equation, we can obtain that the probability density for $P k$ is as following

$$
p k=\frac{m_{0}+\beta t-1}{m} \cdot e^{-\left|m_{0}+\beta t-1 e^{1-\frac{k}{m}}-m_{0}\right|} \cdot e^{-\frac{k}{m}}
$$

By means of the Taylor series of $e^{x}$ and because of $k<<m$ we can obtain the probability density

$$
p k \approx \frac{m_{0}+\beta t-1}{m} \cdot e^{-\frac{k}{m}}
$$

The result is same to the result of $B \& A[1]$, but the coefficient is enriched. Seemly, the characteristic connectivity of the model is $k^{*}=m$. The model indicates that if there does not exist preferential attachment, the scale-free feature about power-law distribution of B\&A model does not appear.

\section{Conclusion}

In order to test and verify the effect of parameter $\beta$ on power-law exponent $r$, we simulate the process of network evolution using computer. The result of simulation is denoted by Fig 1 . The exponential number $\beta$ satisfies $\beta=0.7$ and the slope of the dashed line is $r=2.4$. The similar figure can also be found in the paper[6].

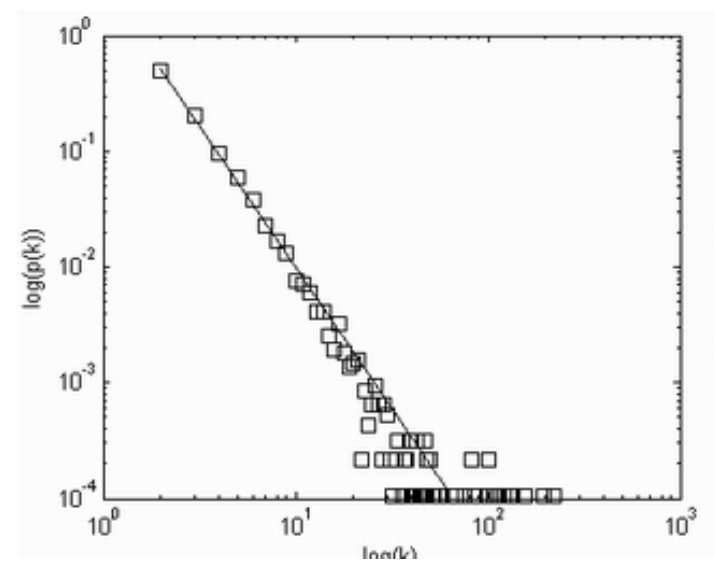

Figure 1 Degree distribution of exponential growth model

From the result of simulation, we can know that the speed $\beta$ of network evolution affect the power-law distribution exponent $r$, following formula $r=2 \beta+1$ basically. Of course, the result does not identical to formula because of the rounding of Taylor series. 
The result of simulation also demonstrates that the scale of local world to which the new vertex preferentially connected is varied dynamically. The size of local world is in direct proportion to the size of network. In fact, network does not evolve according to the fix size of local world in advance.

\section{Acknowledgement}

This work is supported by the humanities and social science planning project of Colleges and universities in Shandong Province No. J13WD07.

\section{References}

[1] Barabási, A.-L., Albert, R., and Jeong, H., Mean-field theory for scale-free random networks, Physica A 272, pp 173-187, 1999.

[2] Barabási, A.-L., Albert, R., Emergence of scaling in random networks, Science 286, pp 509-512 1999.

[3] Albert, R., Jeong, H., and Barabási, A.-L., Diameter of the world-wide web, Nature 401, pp 130-131 1999.

[4] Albert, R. and Barabási, A.-L., Statistical mechanics of complex networks, Rev. Mod. Phys. 74, pp 47-97 2002.

[5] M. E. J. Newman, The structure and function of complex networks. SIAM Review 45, pp 167-256 2003.

[6] Xiang Li, Guanrong Chen. A local-world evolving network model. Physica A 328, 2003 pp 274-286.

[7] Dorogovtsev, S. N., Goltsev, A. V., and Mendes, J. F. F., Pseudofractal scale-free web, Phys. Rev. E 65, 066122, 2002.

[8] Szabó, G., Alava, M., and Kertész, J., Structural transitions in scale-free networks, Preprint cond-mat/0208551, 2002.

[9] Watts, D. J. and Strogatz, S. H., Collective dynamics of 'small-world' networks, Nature 393, pp 440-442 1998.

[10] Ravasz, E. and Barabási, A. L., Hierarchical organization in complex networks, Phys. Rev. E 67, 026112, 2003.

[11] Ravasz, E., Somera, A. L., Mongru, D. A., Oltvai, Z., and Barabási, A. L., Hierarchical organization of modularity in metabolic networks, Science 297, pp 1551-1555, 2002.

[12] Krapivsky, P. L. and Redner, S., Organization of growing random networks, Phys. Rev. E 63, 066123, 2001. 